\title{
Synthesis of new selenophene and thiazole analogues of the Tacrine series
}

\author{
Pierre Seck, ${ }^{a *}$ David Thomae, ${ }^{b}$ Enrico Perspicace, ${ }^{b}$ Stéphanie Hesse, ${ }^{b}$ and Gilbert Kirsch ${ }^{\text {b* }}$ \\ ${ }^{a}$ Laboratoire de Chimie, Faculté des Sciences, de la Technologie et de la Communication, \\ Université du Luxembourg, 162a, avenue de la Faïencerie, L-1511 G.-D. Luxembourg \\ ${ }^{b}$ Laboratoire d'Ingénierie Moléculaire et Biochimie Pharmacologique, Institut Jean Barriol, \\ Université Paul Verlaine - Metz, 1, Boulevard Arago, F-57070 Metz Technopôle, France \\ E-mail: pierre.seck@uni.lu;kirsch@univ-metz.fr
}

Dedicated to Professor Rainer Beckert on the occasion of his 60th birthday

\begin{abstract}
New 2-aminoselenophene-3-carbonitriles and 5-amino-1,3-thiazole-4-carbonitriles were made and reacted with cycloalkanones to give tetrahydroselenolo[2,3-b]quinolines, cycloalkene-fused selenolo[3,2-e]pyridines, [1,3]thiazolo[5,4- $b]$ quinolines, thiazolo[4,5-e]pyridines and tetrahydro$[1,3]$ thiazolo[5,4- $b]$ quinolines.
\end{abstract}

Keywords: 2-aminoselenophene-3-carbonitriles, 5-amino-1,3-thiazole-4-carbonitriles, Gewald reaction, fused pyridines, quinolines, thiazoles, selenophenes

\section{Introduction}

Alzheimer's disease (AD) is characterized by a progressive loss of cognitive functions and has been associated with the impairment of the cholinergic system. Currently, therapeutic intervention still mainly involves the enhancement of the levels of the neurotransmitter acetylcholine (Ach) through the administration of acetylcholinesterase inhibitors. Tacrine (5-amino-1,2,3,4tetrahydroacridine) I (commercial name Cognex ${ }^{\circledR}$ ) belongs to this category of drugs and was authorized in 1993 for AD therapy. Our object was to look at structural modification of Tacrine which may have beneficial biological effects as enhanced acetylcholinesterase inhibition activity and/or diminished hepatotoxicity. We have described already the synthesis of tetrahydrothieno$[3,2-b]$ quinolines $\mathbf{I I},{ }^{1}$ tetrahydrothieno[2,3- $b$ ]quinolines $\mathbf{I I I},{ }^{2}$ tetrahydrothieno[3,4- $\left.b\right]$ quinolines $\mathbf{I V},{ }^{3}$ tetrahydroselenolo[3,2-b]quinolines $\mathbf{V},{ }^{4}$ and tetrahydrothiazolo[5,4- $b$ ]quinolines VI. ${ }^{5}$ (Fig. 1) 
To extend our library of new analogues of Tacrine, we developed methods to prepare tetrahydroselenolo[2,3-b]quinolines and other cycloalkene-fused selenolo[3,2-e]pyridines VII, and tetrahydro[1,3]thiazolo[5,4- $b]$ quinolines and other cycloalkene-fused $[1,3]$ thiazolo[4,5-e]pyridine VIII (Fig. 1).<smiles>Nc1c2c(nc3ccccc13)CCCC2</smiles>

I
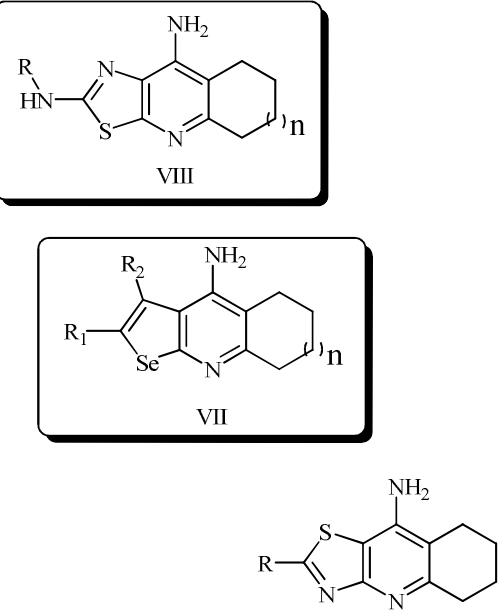

$\mathrm{VI}$<smiles>[R]c1sc2c(N)c3c(nc2c1[R])CCCC3</smiles><smiles>[R]c1sc2nc3c(c(N)c2c1[R])CCCC3</smiles><smiles>[R]c1sc([R])c2c(N)c3c(nc12)CCCC3</smiles><smiles>[R]c1sc2c(N)c3c(nc2c1[R])CCCC3</smiles>

$\mathrm{V}$

Figure 1

\section{Results and discussion}

\section{Synthesis of 2-aminoselenophene-3-carbonitriles}

The precursors of selenoloquinolines and cycloalkene-fused selenolo[3,2-e]pyridines VII are the substituted selenophenes $\mathbf{1}$. They were obtained in one step in good yields using the method described by Gewald. ${ }^{6}$ (Scheme 1)<smiles>[R]CC([R])=O</smiles><smiles>N#CCC#N</smiles>

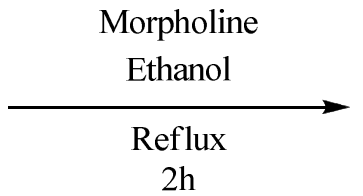<smiles>[R]c1sc(N)c(C#N)c1[R]</smiles>

\section{Scheme 1}


Table 1. Yields obtained in the synthesis of 2-aminoselenophene-3-carbonitriles 1

\begin{tabular}{lll}
\hline Entry & Substituent $^{a}$ & Yield \\
\hline 1a & $\mathrm{R}^{1}=\mathrm{R}^{2}=-\mathrm{CH}_{3}$ & $58 \%$ \\
1b & $-\mathrm{R}^{1}-\mathrm{R}^{2}-=-\left(\mathrm{CH}_{2}\right)_{4^{-}}$ & $73 \%$ \\
1c & $-\mathrm{R}^{1}-\mathrm{R}^{2}-=-\left(\mathrm{CH}_{2}\right)_{5^{-}}$ & $74 \%$ \\
\hline
\end{tabular}

${ }^{a}$ We found it was not possible to obtain 2-aminoselenophene-3-carbonitriles with $\mathrm{R}=p$ methoxyphenyl or $\mathrm{R}=p$-chlorophenyl even in a two-step synthesis

\section{Synthesis of 5-amino-1,3-thiazole-3-carbonitriles}

The precursors of tetrahydro[1,3]thiazolo[5,4- $b]$ quinolines and cycloalkene-fused [1,3]thiazolo[4,5-e]pyridines VIII are the substituted thiazoles $\mathbf{2}$. They were obtained in one step (Scheme 2) in very good yields using methods described by Freeman and $\mathrm{Kim}^{7}$ or Hirota et al. ${ }^{8}$<smiles>Cc1ccc(S(=O)(=O)[O-])cc1</smiles><smiles>[R]N[As]</smiles><smiles>[R]Nc1nc(C#N)c(N)s1</smiles>

2 (a-d)

\section{Scheme 2}

Table 2. Yields obtained in the synthesis of 5-amino-1,3-thiazole-4-carbonitriles 2

\begin{tabular}{ccc}
\hline Entry & Substituent & Yield \\
\hline 2a & $\mathrm{R}=-\mathrm{Ph}$ & $76 \%$ \\
$\mathbf{2 b}$ & $\mathrm{R}=-\mathrm{C}_{6} \mathrm{H}_{4} \mathrm{OMe}$ & $83 \%$ \\
2c & $\mathrm{R}=-\mathrm{C}_{6} \mathrm{H}_{4} \mathrm{Cl}$ & $96 \%$ \\
2d & $\mathrm{R}=-\mathrm{CH}_{2} \mathrm{Ph}$ & $68 \%$ \\
\hline
\end{tabular}

Synthesis of tetrahydroselenopheno[2,3-b]quinolines, cycloalkene-fused selenolo[3,2-e]pyridines, tetrahydro[1,3]thiazolo[5,4-b]quinolines and cycloalkene-fused $[1,3]$ thiazolo[4,5e]pyridines

To prepare these different compounds, we used Friedländer's reaction. ${ }^{9}$ 2-Aminoselenophene-3carbonitriles (1a-c) and 5-amino-1,3-thiazole-4-carbonitriles (2a-d) were reacted with cycloalkanones in dichloroethane in the presence of aluminium chloride. After at least 6 hours of reflux the reaction had come to an end and the expected products (3a-e, Scheme 3, and 4a0-4d2, Scheme 4) were obtained in good or moderate yields depending on the size of the ring of the cycloalkanone used. 
<smiles>[R]c1[se]c(N)c(C#N)c1[R]</smiles>

$1(\mathbf{a}-\mathbf{c})$<smiles>O=C1CCCCC1</smiles><smiles>[R]c1[se]c2nc3c(c(N)c2c1[R])CCCC3</smiles>

$3(\mathbf{a}-\mathbf{e})$

Scheme 3

Table 3. Yields obtained for the synthesis of tetrahydroselenolo[2,3-b]quinolines and cycloalkene-fused selenolo[3,2-e]pyridines (3a-e)

\begin{tabular}{ccc}
\hline Entry & Substituent & Yield \\
\hline 3a $(\mathbf{n}=\mathbf{1})$ & $\mathrm{R}^{1}=\mathrm{R}^{2}=-\mathrm{CH}_{3}$ & $73 \%$ \\
3b $(\mathbf{n}=\mathbf{0})$ & $\mathrm{R}^{1}-\mathrm{R}^{2}=-\left(\mathrm{CH}_{2}\right)_{4^{-}}$ & $83 \%$ \\
3c $(\mathbf{n}=\mathbf{1})$ & $\mathrm{R}^{1}-\mathrm{R}^{2}=-\left(\mathrm{CH}_{2}\right)_{4^{-}}$ & $88 \%$ \\
3d $(\mathbf{n}=\mathbf{2})$ & $\mathrm{R}^{1}-\mathrm{R}^{2}=-\left(\mathrm{CH}_{2}\right)_{4^{-}}$ & $89 \%$ \\
3e $(\mathbf{n}=\mathbf{1})$ & $\mathrm{R}^{1}-\mathrm{R}^{2}=-\left(\mathrm{CH}_{2}\right)_{5^{-}}$ & $86 \%$ \\
\hline
\end{tabular}<smiles>[R]Nc1nc(C#N)c(N)s1</smiles>

\section{Scheme 4}

Table 4. Yields obtained for the synthesis of tetrahydro[1,3]thiazolo[5,4-b]quinolines and cycloalkene-fused $[1,3]$ thiazolo[4,5-e $]$ pyridines (4)

\begin{tabular}{cccc}
\hline \multicolumn{2}{c}{ Entry } & Substituent & Yield \\
\hline $\mathbf{4 a 0}$ & $(\mathbf{n}=\mathbf{0})$ & $\mathrm{R}=$ & $84 \%$ \\
$\mathbf{4 a 1}$ & $(\mathbf{n}=\mathbf{1})$ & $58 \%$ \\
$\mathbf{4 a 2}$ & $\mathbf{( n = 2 )}$ & $84 \%$ \\
\hline $\mathbf{4 b 0}$ & $\mathbf{( n = 0 )}$ & $81 \%$ \\
$\mathbf{4 b 1}$ & $(\mathbf{n = 1})$ & $\mathrm{R}=$ & $35 \%$ \\
$\mathbf{4 b 2}$ & $(\mathbf{n}=\mathbf{2})$ & $87 \%$
\end{tabular}


Table 4 (continued)

\begin{tabular}{llll}
\hline \multicolumn{2}{c}{ Entry } & Substituent & Yield \\
\hline $\mathbf{4 c 0}$ & $(\mathbf{n}=\mathbf{0})$ & $\mathrm{R}=$ & $85 \%$ \\
$\mathbf{4 c 1}$ & $(\mathbf{n}=\mathbf{1})$ & & $33 \%$ \\
$\mathbf{4 c 2}$ & $(\mathbf{n}=\mathbf{2})$ & & $82 \%$ \\
\hline $\mathbf{4 d 0}$ & $(\mathbf{n}=\mathbf{0})$ & $\mathrm{R}=$ & $87 \%$ \\
$\mathbf{4 d 1}$ & $(\mathbf{n}=\mathbf{1})$ & $31 \%$ \\
$\mathbf{4 d 2}$ & $(\mathbf{n}=\mathbf{2})$ & $80 \%$ \\
\hline
\end{tabular}

\section{Conclusions}

Substituted 2-aminoselenophene-3-carbonitriles (1a-c) were obtained in good yields in one step by the Gewald reaction. The difference with the thiophene analogues prepared in ref. 2 is that only very few ketones gave the Gewald reaction with selenium. Substituted 5-amino-1,3thiazole-4-carbonitriles (2a-d) were obtained in good yields in one step following refs 7 or 8 . The substituted 2-aminoselenophene-3-carbonitriles gave substituted tetrahydroselenolo[2,3-b]quinolines and substituted cycloalkene-fused selenolo[3,2-e]pyridines (3a-e) and the substituted 5-amino-1,3-thiazole-3-carbonitriles gave substituted tetrahydro[1,3]thiazolo[5,4- $b]$ quinolines and substituted cycloalkene-fused $[1,3]$ thiazolo[4,5$e$ ]pyridines (4a0-d2) by Friedländer's reaction in one step, the whole synthesis needing only two steps.

\section{Experimental Section}

General Procedures. Melting points were determined on a Stuart SMP3 apparatus and are uncorrected. IR spectra were recorded neat from 4400 to $600 \mathrm{~cm}^{-1}$ on a Perkin Elmer FT-IR Baragon 1000PC equipped with a Graseby-Specac golden gate and treated with the Spectrum (Perkin-Elmer) software 5.3.1. ${ }^{1} \mathrm{H}$ and ${ }^{13} \mathrm{C}$ NMR spectra were recorded on a Bruker AC 250 $\mathrm{MHz}$ spectrometer in $\mathrm{CDCl}_{3}$ or DMSO- $\mathrm{d}_{6}$. The coupling constants are in Hz. HMRS were collected on a Bruker MICROTOF-Q ESI/QqTOF spectrometer.

General procedure to obtain substituted 2-aminoselenophene-3-carbonitriles (1a-c) using Gewald's method ${ }^{\mathbf{6}}$ (products listed in Table 1). The ketone (193 mmol), malonodinitrile (193 mmol), and powdered selenium $193 \mathrm{mg}$-atom) were dissolved / suspended in ethanol (100 ml). 
Diethylamine or morpholine $(100 \mathrm{~mL})$ was added and the mixture was stirred and refluxed over ca. $18 \mathrm{~h}$. After cooling to RT the mixture was filtered through Celite and poured into ice-water. The precipitation was collected by filtration, washed with cold water, dried and purified either by recrystallization from isopropanol or by silica gel column chromatography using dichloromethane as eluent. If there was no precipitation, the mixture was extracted with dichloromethane or diethyl ether. The organic layer was separated, dried on anhydrous sodium sulfate and filtered, and the solvent was evaporated to give the product which was then purified.

2-Amino-4,5-dimethylselenophene-3-carbonitrile (1a). Brownish solid, m.p. 143-145 ${ }^{\circ} \mathrm{C}$ (isopropanol). IR: 3417 and $3323\left(\mathrm{NH}_{2}\right), 2193(\mathrm{CN}), 1717\left(\mathrm{NH}_{2}\right), 1341\left(\mathrm{CH}_{3}\right), 1317\left(\mathrm{NH}_{2}\right), \mathrm{cm}^{-1}$. ${ }^{1} \mathrm{H} \mathrm{NMR}\left(\mathrm{CDCl}_{3}\right): \delta 2.0\left(\mathrm{~s}, 3 \mathrm{H}, \mathrm{CH}_{3}\right), 2.3\left(\mathrm{~s}, 3 \mathrm{H}, \mathrm{CH}_{3}\right), 5.3\left(\mathrm{~s}, 2 \mathrm{H}, \mathrm{NH}_{2}\right) .{ }^{13} \mathrm{C} \mathrm{NMR}\left(\mathrm{DMSO}-d_{6}\right)$ : $\delta 13.6,14.1,86.8,117.6,129.2,158.5,166.2$.

2-Amino-4,5,6,7-tetrahydro-1-benzoselenophene-3-carbonitrile (1b). Red-brownish solid, m.p. 88-90 ${ }^{\circ} \mathrm{C}$. IR: 3420 and $3326\left(\mathrm{NH}_{2}\right), 2190(\mathrm{CN}), 1617\left(\mathrm{NH}_{2}\right), 1431$ and $1379\left(\mathrm{CH}_{2}\right), 1325$ $\left(\mathrm{NH}_{2}\right) \mathrm{cm}^{-1} .{ }^{1} \mathrm{H}$ NMR $\left(\mathrm{CDCl}_{3}\right): \delta 1.75\left(\mathrm{~m}, 4 \mathrm{H}, 2 \times \mathrm{CH}_{2}\right), 2.4\left(\mathrm{t}, 2 \mathrm{H}, \mathrm{CH}_{2}\right), 2.5\left(\mathrm{t}, 2 \mathrm{H}, \mathrm{CH}_{2}\right), 5.0$ $\left(\mathrm{s}, 2 \mathrm{H}, \mathrm{NH}_{2}\right) .{ }^{13} \mathrm{C} \mathrm{NMR}\left(\mathrm{CDCl}_{3}\right): \delta 22.1,23.8,26.0,26.5,90.2,116.4,124.8,133.0,164.8$.

2-Amino-5,6,7,8-tetrahydro-4H-cyclohepta[b]selenophene-3-carbonitrile (1c). Red-brownish solid, m.p. 94-96 ${ }^{\circ} \mathrm{C}$. IR: 3424 and $3329\left(\mathrm{NH}_{2}\right), 2191(\mathrm{CN}) 1615\left(\mathrm{NH}_{2}\right), 1439$ and $1380\left(\mathrm{CH}_{2}\right)$, $1333\left(\mathrm{NH}_{2}\right) \mathrm{cm}^{-1} .{ }^{1} \mathrm{H} \mathrm{NMR}\left(\mathrm{CDCl}_{3}\right): \delta 1.6\left(\mathrm{~m}, 4 \mathrm{H}, 2 \times \mathrm{CH}_{2}\right), 1.8\left(\mathrm{~m}, 2 \mathrm{H}, \mathrm{CH}_{2}\right), 2.6(\mathrm{~m}, 4 \mathrm{H}, 2 \times$ $\left.\mathrm{CH}_{2}\right), 4.9\left(\mathrm{~s}, 2 \mathrm{H}, \mathrm{NH}_{2}\right) .{ }^{13} \mathrm{C} \mathrm{NMR}$ (DMSO- $\left.d_{6}\right): \delta 26.7,27.7,29.7,30.6,31.4,88.0,117.7,123.9$, 136.6, 166.0 .

General procedure to obtain substituted 5-amino-1,3-thiazole-4-carbonitriles (2a-c) using the method of Freeman and $\mathbf{K i m}^{7}$ (products listed in Table 2). Aminomalononitrile tosylate $(9.2 \mathrm{mmol})$ was dissolved in anhydrous 1-methyl-2-pyrrolidinone $(30 \mathrm{~mL})$. Aryl isothiocyanate (9.2 mmol) was added dropwise while stirring. The mixture was stirred for $c a 20 \mathrm{~h}$ at $22-24^{\circ} \mathrm{C}$ under stirring. It was then diluted with 1:1 ethyl acetate / diethyl ether $(150 \mathrm{~mL})$, transferred to a separatory funnel and washed with water $(2 \times 100 \mathrm{~mL})$. The organic layer was separated, dried with anhydrous sodium sulfate and filtered, and the solvent was removed in vacuo. The residue was chromatographed on silica gel with EtOAc / dichloromethane (1:9 to 3:7) to afford the thiazoles.

5-Amino-2-(phenylamino)-1,3-thiazole-4-carbonitrile (2a). Red-brownish solid, m.p. 164$166^{\circ} \mathrm{C}\left(164-165^{7}\right)$ IR: $3691(\mathrm{NH}), 3355$ and $3289\left(\mathrm{NH}_{2}\right), 2198(\mathrm{CN}), 1597\left(\mathrm{NH}_{2}\right), 1575(\mathrm{NH})$, $1345\left(\mathrm{NH}_{2}\right), 743$ and 684 (monosubstituted benzene) $\mathrm{cm}^{-1} .{ }^{1} \mathrm{H}$ NMR (DMSO- $\left.d_{6}\right): \delta 6.1$ (s, 2H, $\left.\mathrm{NH}_{2}\right), 6.8(\mathrm{t}, 1 \mathrm{H}, \mathrm{CH}, J=8.5 \mathrm{~Hz}), 7.1(\mathrm{t}, 2 \mathrm{H}, 2 \times \mathrm{CH}, J=8.5 \mathrm{~Hz}), 7.4(\mathrm{~d}, 2 \mathrm{H}, 2 \times \mathrm{CH}, J=8.5$ $\mathrm{Hz}$ ), 9.3 (s, 1H, NH). ${ }^{13} \mathrm{C}$ NMR (DMSO-d $)_{6}$ ): $\delta$ 95.6, 116.4, 116.6, 120.8, 128.9, 141.0, 147.8, 154.4.

5-Amino-2-[(4-methoxyphenyl)amino]-1,3-thiazole-4-carbonitrile (2b). Brownish solid, m.p. 200-202 ${ }^{\circ} \mathrm{C}\left(199-200^{\circ} \mathrm{C}^{7}\right)$ IR: $3414(\mathrm{NH}), 3324$ and $3171\left(\mathrm{NH}_{2}\right), 2916\left(\mathrm{CH}_{3}\right), 1614\left(\mathrm{NH}_{2}\right), 1583$ 
$(\mathrm{NH}), 1453\left(\mathrm{CH}_{3}\right), 1357\left(\mathrm{NH}_{2}\right), 753$ (disubstituted benzene) $\mathrm{cm}^{-1} .{ }^{1} \mathrm{H}$ NMR (DMSO- $\left.d_{6}\right): \delta 3.7(\mathrm{~s}$, $\left.3 \mathrm{H}, \mathrm{OCH}_{3}\right), 6.7\left(\mathrm{~s}, 2 \mathrm{H}, \mathrm{NH}_{2}\right), 6.8(\mathrm{~d}, 2 \mathrm{H}, 2 \times \mathrm{CH}, J=8.5 \mathrm{~Hz}), 7.4(\mathrm{~d}, 2 \mathrm{H}, 2 \times \mathrm{CH}, J=8.5 \mathrm{~Hz})$, $9.5(\mathrm{~s}, 1 \mathrm{H}, \mathrm{NH}) .{ }^{13} \mathrm{C} \mathrm{NMR}\left(\mathrm{DMSO}-d_{6}\right): \delta 55.1,95.6,114.1,115.7,116.7,118.0,134.5,148.7$, 153.8 .

5-Amino-2-[(4-chlorophenyl)amino]-1,3-thiazole-4-carbonitrile (2c). Brownish solid, m.p. 205-208 ${ }^{\circ} \mathrm{C}\left(210-211^{\circ} \mathrm{C}^{7}\right)$ IR: $3366(\mathrm{NH}), 3307$ and $3178\left(\mathrm{NH}_{2}\right), 2199(\mathrm{CN}), 1600\left(\mathrm{NH}_{2}\right), 1573$ $(\mathrm{NH}), 1320\left(\mathrm{NH}_{2}\right), 746$ (disubstituted benzene) $\mathrm{cm}^{-1} .{ }^{1} \mathrm{H}$ NMR (DMSO- $\left.d_{6}\right): \delta 6.8\left(\mathrm{~s}, 2 \mathrm{H}, \mathrm{NH}_{2}\right)$, $7.4(\mathrm{~d}, 2 \mathrm{H}, 2 \mathrm{xCH}, \mathrm{J}=7.8 \mathrm{~Hz}), 7.6(\mathrm{~d}, 2 \mathrm{H}, 2 \mathrm{xCH}, \mathrm{J}=7.8 \mathrm{~Hz}), 9.9(\mathrm{~s}, 1 \mathrm{H}, \mathrm{NH}) .{ }^{13} \mathrm{C} \mathrm{NMR}$ (DMSO- $d_{6}$ ): $\delta$ 95.6, 116.6, 117.8, 124.1, 128.7, 139.8, 147.4, 154.6.

Procedure to obtain substituted-5-amino-1,3-thiazole-4-carbonitrile (2d) using the method of Hirota $\boldsymbol{e t} \boldsymbol{a l}^{\mathbf{8}} \mathrm{N}, \mathrm{N}$-Diisopropylethylamine $(6.32 \mathrm{mmol})$ was added to a suspension of aminomalononitrile $p$-toluenesulfonate $(7.9 \mathrm{mmol})$ in dry THF $(20 \mathrm{~mL})$, and the mixture was stirred under Ar at RT for $5 \mathrm{~min}$. Benzyl thiocyanate $(15.79 \mathrm{mmol})$ in dry THF was added and the mixture was stirred at $c a 40^{\circ} \mathrm{C}$ for 72 hours. The solvent was removed under reduced pressure and the residue was partitioned between EtOAc $(100 \mathrm{~mL})$ and water $(100 \mathrm{~mL})$. The organic layer was washed with brine $(50 \mathrm{~mL})$ and dried over anhydrous sodium sulfate. The solvent was evaporated and the residue was chromatographed on silica gel with EtOAc / dichloromethane (3:7).

5-Amino-2-(benzylamino)-1,3-thiazole-4-carbonitrile (2d). Red-brownish solid, m.p. 142144 ${ }^{\circ} \mathrm{C}$. IR: $3406(\mathrm{NH}), 3185$ and $3109\left(\mathrm{NH}_{2}\right), 2906\left(\mathrm{CH}_{2}\right), 2209(\mathrm{CN}), 1609\left(\mathrm{NH}_{2}\right), 1546(\mathrm{NH})$, $1453\left(\mathrm{CH}_{2}\right), 1325\left(\mathrm{NH}_{2}\right), 756$ and 689 (monosubstituted benzene) $\mathrm{cm}^{-1} .{ }^{1} \mathrm{H}$ NMR (DMSO- $\left.d_{6}\right): \delta$ $4.3\left(\mathrm{~d}, 2 \mathrm{H}, \mathrm{CH}_{2}\right), 6.45\left(\mathrm{~s}, 2 \mathrm{H}, \mathrm{NH}_{2}\right), 7.3(\mathrm{~m}, 5 \mathrm{H}$, monosubstituted benzene), 7.55 (s, 1H, NH). ${ }^{13} \mathrm{C}$ NMR (DMSO- $d_{6}$ ): $\delta 46.7,95.8,116.9,126.9,127.42,127.45,139.1,153.4,153.6$. HRMS (APCI) calcd for $\mathrm{C}_{11} \mathrm{H}_{11} \mathrm{~N}_{4} \mathrm{~S}[\mathrm{M}+\mathrm{H}]^{+} 231.0699$, found 231.0705 .

General procedure to obtain substituted tetrahydroselenopheno[2,3-b]quinolines and cycloalkeno[b]selenopheno[3,2-e]pyridines (3a-3e, Table 3), substituted tetrahydro[1,3]thiazolo[5,4- $b]$ quinolines and substituted cycloalkeno $[b]\{1,3]$ thiazolo[4,5-e $]$ pyridines (4a0 4d2, Table 4) by Friedländer reaction. Aluminum chloride (17 $\mathrm{mmol}$ for the selenophenes 1 and $34 \mathrm{mmol}$ for the thiazoles 2) was suspended in dry 1,2-dichloroethane (10 $\mathrm{mL}$ per mmol of aluminium chloride) at RT under Ar. The selenophene or thiazole $(10 \mathrm{mmol})$ and ketone were added and the reaction mixture was heated under reflux for a minimum of $6 \mathrm{~h}$. When the reaction was completed (monitoring by TLC), a mixture of THF / water (2:1) was added at RT and sodium hydroxide was added until the solution became alkaline. After stirring for at least 30 minutes, the mixture was extracted with dichloromethane $(3 \times 30 \mathrm{~mL})$. The combined organic layers were dried over anhydrous sodium sulfate and solvent was evaporated to give the crude product which was purified by silica gel chromatography using dichloromethane (100\%) for the selenopheno derivatives and EtOAc / dichloromethane (1:9 to 5:5) for the thiazolo derivatives. 
2,3-Dimethyl-5,6,7,8-tetrahydroselenolo[2,3-b]quinolin-4-amine (3a). Brownish solid, m.p. 114-116 ${ }^{\circ} \mathrm{C}$. IR: 3357 and $3290\left(\mathrm{NH}_{2}\right), 2926$ and $2855\left(\mathrm{CH}_{3}\right.$ and $\left.\mathrm{CH}_{2}\right), 1617\left(\mathrm{NH}_{2}\right), 1267\left(\mathrm{NH}_{2}\right)$ $\mathrm{cm}^{-1} .{ }^{1} \mathrm{H} \mathrm{NMR}\left(\mathrm{CDCl}_{3}\right): \delta 1.8\left(\mathrm{~m}, 4 \mathrm{H}, 2 \times \mathrm{CH}_{2}\right), 2.3\left(\mathrm{~m}, 6 \mathrm{H}, 2 \times \mathrm{CH}_{3}\right), 2.5\left(\mathrm{~m}, 2 \mathrm{H}, \mathrm{CH}_{2}\right), 2.8(\mathrm{~m}$, $\left.2 \mathrm{H}, \mathrm{CH}_{2}\right), 4.7\left(\mathrm{~s}, 2 \mathrm{H}, \mathrm{NH}_{2}\right) .{ }^{13} \mathrm{C} \mathrm{NMR}\left(\mathrm{CDCl}_{3}\right): \delta 22.6,23.1,26.8,31.9,42.0,112.0,121.4$, 126.2, 132.3, 132.6, 148.3, 153.0, 160.2. HRMS (ESI) calcd for $\mathrm{C}_{13} \mathrm{H}_{17} \mathrm{~N}_{2} \mathrm{Se}[\mathrm{M}+\mathrm{H}]^{+} 281.0552$, found 281.0548 .

2,3,6,7,8,9-Hexahydro-1H-cyclopenta[b][1]benzoselenolo[3,2-e]pyridin-10-amine

(3b). Beige solid, m.p. 198-200 ${ }^{\circ}$ C. IR: 3501 and $3295\left(\mathrm{NH}_{2}\right), 2932$ and $2829\left(\mathrm{CH}_{2}\right), 1638\left(\mathrm{NH}_{2}\right), 1448$ and $1398\left(\mathrm{CH}_{2}\right), 1275\left(\mathrm{NH}_{2}\right) \mathrm{cm}^{-1} .{ }^{1} \mathrm{H} \mathrm{NMR}\left(\mathrm{CDCl}_{3}\right): \delta 1.8\left(\mathrm{~m}, 4 \mathrm{H}, 2 \times \mathrm{CH}_{2}\right), 2.1\left(\mathrm{~m}, 2 \mathrm{H}, \mathrm{CH}_{2}\right)$,

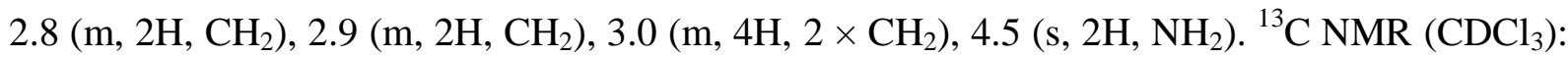
$\delta$ 22.6, 22.8, 22.9, 27.0, 28.1, 28.4, 34.1, 117.1, 120.5, 128.7, 135.3, 146.2, 162.1, 163.8. HRMS (ESI) calcd for $\mathrm{C}_{14} \mathrm{H}_{17} \mathrm{~N}_{2} \mathrm{Se}[\mathrm{M}+\mathrm{H}]^{+} 293.0552$, found 293.0558 .

1,2,3,4,7,8,9,10-Octahydro[1] benzoselenolo[2,3-b]quinolin-11-amine (3c). Orange solid, m.p. 206-208 ${ }^{\circ} \mathrm{C}$. IR: 3470 and $3370\left(\mathrm{NH}_{2}\right), 2849$ and $2923\left(\mathrm{CH}_{2}\right), 1612\left(\mathrm{NH}_{2}\right), 1430\left(\mathrm{CH}_{2}\right), 1273$ $\left(\mathrm{NH}_{2}\right) \mathrm{cm}^{-1} .{ }^{1} \mathrm{H} \mathrm{NMR}\left(\mathrm{CDCl}_{3}\right): \delta 1.8\left(\mathrm{~m}, 6 \mathrm{H}, 3 \times \mathrm{CH}_{2}\right), 2.35\left(\mathrm{~m}, 4 \mathrm{H}, 2 \times \mathrm{CH}_{2}\right), 2.8(\mathrm{~m}, 4 \mathrm{H}, 2 \times$ $\left.\mathrm{CH}_{2}\right), 2.9\left(\mathrm{~m}, 2 \mathrm{H}, \mathrm{CH}_{2}\right), 4.5\left(\mathrm{~s}, 2 \mathrm{H}, \mathrm{NH}_{2}\right) .{ }^{13} \mathrm{C} \mathrm{NMR}\left(\mathrm{CDCl}_{3}\right): \delta 22.7,22.85,22.89,23.0,28.1$, 28.4, 32.9, 42.0, 111.8, 120.4, 128.6, 135.3, 147.8, 153.6, 161.8. HRMS (ESI) calcd for $\mathrm{C}_{15} \mathrm{H}_{19} \mathrm{~N}_{2} \mathrm{Se}[\mathrm{M}+\mathrm{H}]^{+} 307.0708$, found 307.0732.

1,2,3,4,8,9,10,11-Octahydro-7H-cyclohepta[b][1]benzoselenolo[3,2-e]pyridin-12-amine (3d). Brownish solid, m.p. 222-224 ${ }^{\circ} \mathrm{C}$. IR: 3505 and $3295\left(\mathrm{NH}_{2}\right), 2908$ and $2842\left(\mathrm{CH}_{2}\right), 1632\left(\mathrm{NH}_{2}\right)$, $1429\left(\mathrm{CH}_{2}\right), 1271\left(\mathrm{NH}_{2}\right) \mathrm{cm}^{-1} .{ }^{1} \mathrm{H}$ NMR $\left(\mathrm{CDCl}_{3}\right): \delta 1.8\left(\mathrm{~m}, 6 \mathrm{H}, 3 \times \mathrm{CH}_{2}\right), 2.1\left(\mathrm{~m}, 4 \mathrm{H}, 2 \times \mathrm{CH}_{2}\right)$, $2.7\left(\mathrm{~m}, 2 \mathrm{H}, \mathrm{CH}_{2}\right), 3.05\left(\mathrm{~m}, 2 \mathrm{H}, \mathrm{CH}_{2}\right), 3.2\left(\mathrm{~m}, 4 \mathrm{H}, 2 \times \mathrm{CH}_{2}\right), 4.9\left(\mathrm{~s}, 2 \mathrm{H}, \mathrm{NH}_{2}\right) .{ }^{13} \mathrm{C} \mathrm{NMR}$ $\left(\mathrm{CDCl}_{3}\right): \delta 22.8,25.3,26.5,27.0,28.2,28.6,30.3,32.1,38.5,117.4,121.4,128.7,136.4,147.2$, 159.8, 160.7. HRMS (ESI) calcd for $\mathrm{C}_{16} \mathrm{H}_{21} \mathrm{~N}_{2} \mathrm{Se}[\mathrm{M}+\mathrm{H}]^{+}$321.0865, found 321.0874.

1,2,3,48,9,10,11-Octahydro-7H-cyclohepta[4,5]selenolo[2,3-b]quinolin-12-amine

(3e). Orange solid, m.p. 176-178 ${ }^{\circ}$ C. IR: 3501 and $3299\left(\mathrm{NH}_{2}\right), 2913$ and $2846\left(\mathrm{CH}_{2}\right), 1632\left(\mathrm{NH}_{2}\right)$, $1428\left(\mathrm{CH}_{2}\right), 1278\left(\mathrm{NH}_{2}\right) \mathrm{cm}^{-1} .{ }^{1} \mathrm{H}$ NMR $\left(\mathrm{CDCl}_{3}\right): \delta 1.6\left(\mathrm{~m}, 6 \mathrm{H}, 3 \times \mathrm{CH}_{2}\right), 1.8\left(\mathrm{~m}, 6 \mathrm{H}, 3 \times \mathrm{CH}_{2}\right)$, $2.4\left(\mathrm{~m}, 4 \mathrm{H}, 2 \times \mathrm{CH}_{2}\right), 2.8\left(\mathrm{~m}, 2 \mathrm{H}, \mathrm{CH}_{2}\right), 4.6\left(\mathrm{~s}, 2 \mathrm{H}, \mathrm{NH}_{2}\right) .{ }^{13} \mathrm{C} \mathrm{NMR}\left(\mathrm{CDCl}_{3}\right): \delta 22.7,23.1,26.1$, 27.0, 27.3, 30.6, 30.9, 32.7, 112.2, 122.1, 132.6, 133.6, 140.3, 147.7, 153.3, 161.2. HRMS (ESI) calcd for $\mathrm{C}_{16} \mathrm{H}_{21} \mathrm{~N}_{2} \mathrm{Se}[\mathrm{M}+\mathrm{H}]^{+} 321.0865$, found 321.0866 .

$N^{2}$-Phenyl-6,7-dihydro-5H-cyclopenta $[\boldsymbol{b}][1,3]$ thiazolo[4,5-e $]$ pyridine-2,8-diamine (4a0). Orange solid, m.p. 248-250 ${ }^{\circ}$ C. IR: $3494(\mathrm{NH}), 3388$ and $3241\left(\mathrm{NH}_{2}\right), 2954\left(\mathrm{CH}_{2}\right), 1597\left(\mathrm{NH}_{2}\right)$, $1589(\mathrm{NH}), 1443\left(\mathrm{CH}_{2}\right), 1342\left(\mathrm{NH}_{2}\right), 687$ and 742 (monosubstituted benzene) $\mathrm{cm}^{-1} .{ }^{1} \mathrm{H}$ NMR (DMSO- $\left.d_{6}\right): \delta 2.8\left(\mathrm{~m}, 4 \mathrm{H}, 2 \times \mathrm{CH}_{2}\right), 3.3\left(\mathrm{~m}, 2 \mathrm{H}, \mathrm{CH}_{2}\right), 6.0\left(\mathrm{~s}, 2 \mathrm{H}, \mathrm{NH}_{2}\right), 7.0(\mathrm{t}, 1 \mathrm{H}, \mathrm{CH}, J=8.5$ $\mathrm{Hz}), 7.3(\mathrm{t}, 2 \mathrm{H}, 2 \times \mathrm{CH}, J=8.5 \mathrm{~Hz}), 7.8(\mathrm{~d}, 2 \mathrm{H}, 2 \times \mathrm{CH}, J=8.5 \mathrm{~Hz}), 10.2(\mathrm{~s}, 1 \mathrm{H}, \mathrm{NH}) .{ }^{13} \mathrm{C} \mathrm{NMR}$ (DMSO- $d_{6}$ ): $\delta 22.2,22.6,23.2,32.5,112.0,117.4,121.4,128.9,130.3,140.8,142.9,149.6$, 151.4, 156.1. HRMS (ESI) calcd for $\mathrm{C}_{15} \mathrm{H}_{15} \mathrm{~N}_{4} \mathrm{~S}[\mathrm{M}+\mathrm{H}]^{+} 283.1012$, found 283.1005.

$\boldsymbol{N}^{\mathbf{2}}$-Phenyl-5,6,7,8-tetrahydro[1,3]thiazolo[5,4-b]quinoline-2,9-diamine (4a1). Beige solid, m.p. $200-202^{\circ} \mathrm{C}$. IR: 3395 and $3214\left(\mathrm{NH}_{2}\right), 2359\left(\mathrm{CH}_{2}\right), 1588\left(\mathrm{NH}_{2}\right), 1537(\mathrm{NH}), 1445\left(\mathrm{CH}_{2}\right)$, $1312\left(\mathrm{NH}_{2}\right), 722$ and 688 (monosubstituted benzene) $\mathrm{cm}^{-1}$ ). ${ }^{1} \mathrm{H}$ NMR (DMSO- $\left.d_{6}\right): \delta 1.8(\mathrm{~m}, 4 \mathrm{H}$, 
$\left.2 \times \mathrm{CH}_{2}\right), 2.4\left(\mathrm{~m}, 2 \mathrm{H}, \mathrm{CH}_{2}\right), 2.6\left(\mathrm{~m}, 2 \mathrm{H}, \mathrm{CH}_{2}\right), 5.8\left(\mathrm{~s}, 2 \mathrm{H}, \mathrm{NH}_{2}\right), 7.0(\mathrm{t}, 1 \mathrm{H}, \mathrm{CH}, J=8.5 \mathrm{~Hz}), 7.3$ $(\mathrm{t}, 2 \mathrm{H}, 2 \times \mathrm{CH}, J=8.5 \mathrm{~Hz}), 7.8(\mathrm{~d}, 2 \mathrm{H}, 2 \times \mathrm{CH}, J=8.5 \mathrm{~Hz}), 10.2(\mathrm{~s}, 1 \mathrm{H}, \mathrm{NH}) .{ }^{13} \mathrm{C} \mathrm{NMR}$ $\left(\mathrm{DMSO}-d_{6}\right): \delta 22.2,22.6,23.2,32.5,112.1,117.4,121.4,128.9,130.4,140.7,142.9,149.6$, 151.4, 156.2. HRMS (ESI) calcd for $\mathrm{C}_{16} \mathrm{H}_{17} \mathrm{~N}_{4} \mathrm{~S}[\mathrm{M}+\mathrm{H}]^{+} 297.1168$, found 297.1160.

$N^{2}$-Phenyl-6,7,8,9-tetrahydro-5H-cyclohepta[b][1,3]thiazolo[4,5-e $]$ pyridine-2,10-diamine (4a2). Beige solid, m.p. 212-214 ${ }^{\circ} \mathrm{C}$. IR: $3496(\mathrm{NH}), 3392$ and $3257\left(\mathrm{NH}_{2}\right), 2909\left(\mathrm{CH}_{2}\right), 1587$ $\left(\mathrm{NH}_{2}\right), 1529(\mathrm{NH}), 1444\left(\mathrm{CH}_{2}\right), 1310\left(\mathrm{NH}_{2}\right), 746$ and 687 (monosubstituted benzene) $\mathrm{cm}^{-1} .{ }^{1} \mathrm{H}$ NMR (DMSO- $\left.d_{6}\right): \delta 1.5\left(\mathrm{~m}, 4 \mathrm{H}, 2 \times \mathrm{CH}_{2}\right), 1.7\left(\mathrm{~m}, 2 \mathrm{H}, \mathrm{CH}_{2}\right), 2.6\left(\mathrm{~m}, 2 \mathrm{H}, \mathrm{CH}_{2}\right), 2.7(\mathrm{~m}, 2 \mathrm{H}$, $\left.\mathrm{CH}_{2}\right), 5.8\left(\mathrm{~s}, 2 \mathrm{H}, \mathrm{NH}_{2}\right), 6.8(\mathrm{t}, 1 \mathrm{H}, \mathrm{CH}, J=8.5 \mathrm{~Hz}), 7.2(\mathrm{t}, 2 \mathrm{H}, 2 \times \mathrm{CH}, J=8.5 \mathrm{~Hz})$, 7.7. (d, $2 \mathrm{H}, 2$ $\times \mathrm{CH}, J=8.5 \mathrm{~Hz}), 10.1(\mathrm{~s}, 1 \mathrm{H}, \mathrm{NH}) .{ }^{13} \mathrm{C} \mathrm{NMR}\left(\mathrm{DMSO}-d_{6}\right): \delta 20.6,25.1,26.5,27.3,31.8,117.0$, 117.3, 121.4, 128.8, 131.4, 140.8, 142.4, 148.4, 156.7, 158.5. HRMS (ESI) calcd for $\mathrm{C}_{17} \mathrm{H}_{19} \mathrm{~N}_{4} \mathrm{~S}$ $[\mathrm{M}+\mathrm{H}]^{+} 311.1325$, found 311.1332 .

$\boldsymbol{N}^{\mathbf{2}}$-(4-Methoxyphenyl)-6,7-dihydro-5H-cyclopenta[b][1,3]thiazolo[4,5-e $]$ pyridine-2,8-diamine (4b0). Orange solid, m.p. 205-207 ${ }^{\circ} \mathrm{C}$. IR: $3448(\mathrm{NH}), 3377$ and $3187\left(\mathrm{NH}_{2}\right), 2899\left(\mathrm{CH}_{3}\right.$ and $\left.\mathrm{CH}_{2}\right), 1598\left(\mathrm{NH}_{2}\right), 1534(\mathrm{NH}), 1452\left(\mathrm{CH}_{3}\right), 1382\left(\mathrm{NH}_{2}\right), 751$ (disubstituted benzene) $\mathrm{cm}^{-1}$. ${ }^{1} \mathrm{H}$ NMR (DMSO- $\left.d_{6}\right): \delta 2.0\left(\mathrm{~m}, 2 \mathrm{H}, \mathrm{CH}_{2}\right), 2.7\left(\mathrm{~m}, 4 \mathrm{H}, 2 \times \mathrm{CH}_{2}\right), 3.7\left(\mathrm{~s}, 3 \mathrm{H}, \mathrm{OCH}_{3}\right), 5.9(\mathrm{~s}, 2 \mathrm{H}$, $\left.\mathrm{NH}_{2}\right), 6.8(\mathrm{~d}, 2 \mathrm{H}, 2 \times \mathrm{CH}, J=8.2 \mathrm{~Hz}), 7.7 .(\mathrm{d}, 2 \mathrm{H}, 2 \times \mathrm{CH}, J=8.2 \mathrm{~Hz}), 10.0(\mathrm{~s}, 1 \mathrm{H}, \mathrm{NH}) .{ }^{13} \mathrm{C}$ NMR (DMSO-d $d_{6}$ : $\delta$ 22.6, 27.2, 33.6, 45.1, 114.0, 116.3, 119.0, 130.6, 134.4, 141.3, 151.1, 154.0, 156.5, 158.6, 159.1. HRMS (ESI) calcd for $\mathrm{C}_{16} \mathrm{H}_{17} \mathrm{ON}_{4} \mathrm{~S}[\mathrm{M}+\mathrm{H}]^{+}$313.1118, found 313.1109 .

$\boldsymbol{N}^{\mathbf{2}}$-(4-Methoxyphenyl)-5,6,7,8-tetrahydro[1,3]thiazolo[5,4-b]quinoline-2,9-diamine (4b1). Beige solid, m.p. 195-197 ${ }^{\circ} \mathrm{C}$. IR: $3475(\mathrm{NH}), 3370$ and $3186\left(\mathrm{NH}_{2}\right), 2920\left(\mathrm{CH}_{3}\right.$ and $\left.\mathrm{CH}_{2}\right), 1594$ $\left(\mathrm{NH}_{2}\right), 1535(\mathrm{NH}), 1462\left(\mathrm{CH}_{3}\right), 1311\left(\mathrm{NH}_{2}\right), 752$ (disubstituted benzene) $\mathrm{cm}^{-1} .{ }^{1} \mathrm{H}$ NMR (DMSO- $d_{6}$ ): $\delta 1.7\left(\mathrm{~m}, 4 \mathrm{H}, 2 \times \mathrm{CH}_{2}\right), 2.5\left(\mathrm{~m}, 2 \mathrm{H}, \mathrm{CH}_{2}\right), 2.7\left(\mathrm{~m}, 2 \mathrm{H}, \mathrm{CH}_{2}\right), 3.7\left(\mathrm{~s}, 3 \mathrm{H}, \mathrm{OCH}_{3}\right), 5.7$ $\left(\mathrm{s}, 2 \mathrm{H}, \mathrm{NH}_{2}\right), 6.9(\mathrm{~d}, 2 \mathrm{H}, 2 \times \mathrm{CH}, J=8.2 \mathrm{~Hz}), 7.7(\mathrm{~d}, 2 \mathrm{H}, 2 \times \mathrm{CH}, J=8.2 \mathrm{~Hz}), 10.0(\mathrm{~s}, 1 \mathrm{H}, \mathrm{NH})$. ${ }^{13} \mathrm{C}$ NMR (DMSO- $\left.d_{6}\right): \delta 22.2,22.6,23.2,32.5,55.1,112.1,114.1,119.1,130.5,134.3,142.6$, 149.5, 151.1, 154.1, 156.6. HRMS (ESI) calcd for $\mathrm{C}_{17} \mathrm{H}_{19} \mathrm{ON}_{4} \mathrm{~S}[\mathrm{M}+\mathrm{H}]^{+} 327.1279$, found 327.1274 .

$N^{2}$-(4-Methoxyphenyl)-6,7,8,9-tetrahydro-5H-cyclohepta[b][1,3]thiazolo[4,5-e $]$ pyridine2,10-diamine (4b2). Beige solid, m.p. 179-181 ${ }^{\circ} \mathrm{C}$. IR: $3481(\mathrm{NH}), 3372$ and $3184\left(\mathrm{NH}_{2}\right), 2914$ $\left(\mathrm{CH}_{3}\right.$ and $\left.\mathrm{CH}_{2}\right), 1585\left(\mathrm{NH}_{2}\right), 1534(\mathrm{NH}) 1443\left(\mathrm{CH}_{3}\right), 1310\left(\mathrm{NH}_{2}\right), 723$ (disubstituted benzene) $\mathrm{cm}^{-1} .{ }^{1} \mathrm{H}$ NMR (DMSO-d $): \delta 1.5\left(\mathrm{~m}, 4 \mathrm{H}, 2 \times \mathrm{CH}_{2}\right), 1.7\left(\mathrm{~m}, 2 \mathrm{H}, \mathrm{CH}_{2}\right), 2.6\left(\mathrm{~m}, 2 \mathrm{H}, \mathrm{CH}_{2}\right), 2.8(\mathrm{~m}$, $\left.2 \mathrm{H}, \mathrm{CH}_{2}\right), 3.7\left(3 \mathrm{H}, \mathrm{OCH}_{3}\right), 5.8\left(\mathrm{~s}, 2 \mathrm{H}, \mathrm{NH}_{2}\right), 6.9(\mathrm{~d}, 2 \mathrm{H}, 2 \times \mathrm{CH}, J=8.2 \mathrm{~Hz}), 7.8(\mathrm{~d}, 2 \mathrm{H}, 2 \times$ $\mathrm{CH}, J=8.2 \mathrm{~Hz}$ ), $10.1(\mathrm{~s}, 1 \mathrm{H}, \mathrm{NH}) .{ }^{13} \mathrm{C} \mathrm{NMR}$ (DMSO- $\left.d_{6}\right): \delta 20.7,24.8,26.5,27.3,31.9,55.1$, $114.0,117.3,118.7,131.5,134.2,142.1,148.2,154.2,157.2,158.1$. HRMS (ESI) calcd for $\mathrm{C}_{18} \mathrm{H}_{21} \mathrm{~N}_{4} \mathrm{OS}[\mathrm{M}+\mathrm{H}]^{+} 341.1431$, found 341.1454.

$N^{2}$-(4-Chlorophenyl)-6,7-dihydro-5H-cyclopenta[b][1,3] thiazolo[4,5-e $]$ pyridine-2,8-diamine (4c0). Red-brownish solid, m.p. 294-296 ${ }^{\circ} \mathrm{C}$. IR: $3483(\mathrm{NH}), 3382$ and $3296\left(\mathrm{NH}_{2}\right), 2892\left(\mathrm{CH}_{2}\right)$, $1594\left(\mathrm{NH}_{2}\right), 1538(\mathrm{NH}), 1487\left(\mathrm{CH}_{2}\right), 753$ (disubstituted benzene) $\mathrm{cm}^{-1} .{ }^{1} \mathrm{H}$ NMR (DMSO- $\left.d_{6}\right): \delta$ $2.0\left(\mathrm{~m}, 2 \mathrm{H}, \mathrm{CH}_{2}\right), 2.7\left(\mathrm{~m}, 4 \mathrm{H}, 2 \times \mathrm{CH}_{2}\right), 6.0\left(\mathrm{~s}, 2 \mathrm{H}, \mathrm{NH}_{2}\right), 7.3(\mathrm{~d}, 2 \mathrm{H}, 2 \times \mathrm{CH}, J=8.2 \mathrm{~Hz}), 7.9(\mathrm{~d}$, 
$2 \mathrm{H}, 2 \times \mathrm{CH}, J=8.2 \mathrm{~Hz}), 10.3(\mathrm{~s}, 1 \mathrm{H}, \mathrm{NH}) .{ }^{13} \mathrm{C} \mathrm{NMR}\left(\mathrm{DMSO}-d_{6}\right): \delta 22.6,27.3,33.6,116.5$, $119.0,124.6,128.6,130.3,139.7,141.7,151.2,155.7,160.1$. HRMS (ESI) calcd for $\mathrm{C}_{15} \mathrm{H}_{14} \mathrm{ClN}_{4} \mathrm{~S}[\mathrm{M}+\mathrm{H}]^{+} 317.0628$, found 317.0622 .

$\boldsymbol{N}^{\mathbf{2}}$-(4-Chlorophenyl)-5,6,7,8-tetrahydro[1,3]thiazolo[5,4-b]quinoline-2,9-diamine (4c1). Orange solid, m.p. $260-262^{\circ} \mathrm{C}$. IR: $3486(\mathrm{NH}), 3385$ and $3249\left(\mathrm{NH}_{2}\right), 2922\left(\mathrm{CH}_{2}\right), 1590\left(\mathrm{NH}_{2}\right)$, $1537(\mathrm{NH}), 1486\left(\mathrm{CH}_{2}\right), 1310\left(\mathrm{NH}_{2}\right), 722$ (disubstituted benzene) $\mathrm{cm}^{-1} .{ }^{1} \mathrm{H}$ NMR (DMSO- $\left.d_{6}\right): \delta$ $1.6\left(\mathrm{~m}, 4 \mathrm{H}, 2 \times \mathrm{CH}_{2}\right), 1.8\left(\mathrm{~m}, 2 \mathrm{H}, \mathrm{CH}_{2}\right), 2.5\left(\mathrm{~m}, 2 \mathrm{H}, \mathrm{CH}_{2}\right), 5.7\left(\mathrm{~s}, 2 \mathrm{H}, \mathrm{NH}_{2}\right), 7.1(\mathrm{~d}, 2 \mathrm{H}, 2 \times \mathrm{CH}$, $J=8.2 \mathrm{~Hz}), 7.7 .(\mathrm{d}, 2 \mathrm{H}, 2 \times \mathrm{CH}, J=8.2 \mathrm{~Hz}), 10.2(\mathrm{~s}, 1 \mathrm{H}, \mathrm{NH}) .{ }^{13} \mathrm{C}$ NMR $\left(\mathrm{DMSO}-d_{6}\right): \delta 20.7$, 22.0, 23.2, 32.5, 112.1, 119.1, 124.7, 128.6, 130.1, 139.7, 143.1, 149.7, 151.6, 155.8. HRMS (ESI) calcd for $\mathrm{C}_{16} \mathrm{H}_{16} \mathrm{ClN}_{4} \mathrm{~S}[\mathrm{M}+\mathrm{H}]^{+} 331.0772$, found 331.0779 .

$\boldsymbol{N}^{\mathbf{2}}$-(4-Chlorophenyl)-6,7,8,9-tetrahydro-5 $\boldsymbol{H}$-cyclohepta[b][1,3]thiazolo[4,5-e $]$ pyridine-2,10-

diamine (4c2). Red-brownish solid, m.p. 272-274 ${ }^{\circ} \mathrm{C}$. IR: $3483(\mathrm{NH}), 3368$ and $3182\left(\mathrm{NH}_{2}\right)$, $2920\left(\mathrm{CH}_{2}\right), 1583\left(\mathrm{NH}_{2}\right), 1528(\mathrm{NH}), 1488\left(\mathrm{CH}_{2}\right), 1308\left(\mathrm{NH}_{2}\right), 735$ (disubstituted benzene) $\mathrm{cm}^{-1}$. ${ }^{1} \mathrm{H}$ NMR (DMSO- $\left.d_{6}\right): \delta 1.5\left(\mathrm{~m}, 4 \mathrm{H}, 2 \times \mathrm{CH}_{2}\right), 1.6\left(\mathrm{~m}, 2 \mathrm{H}, \mathrm{CH}_{2}\right), 2.6\left(\mathrm{~m}, 2 \mathrm{H}, \mathrm{CH}_{2}\right), 2.8(\mathrm{~m}, 2 \mathrm{H}$, $\left.\mathrm{CH}_{2}\right), 5.7\left(\mathrm{~s}, 2 \mathrm{H}, \mathrm{NH}_{2}\right), 7.1(\mathrm{~d}, 2 \mathrm{H}, 2 \times \mathrm{CH}, J=8.2 \mathrm{~Hz}), 7.7(\mathrm{~d}, 2 \mathrm{H}, 2 \times \mathrm{CH}, J=8.2 \mathrm{~Hz}), 10.2(\mathrm{~s}$, $1 \mathrm{H}, \mathrm{NH}) .{ }^{13} \mathrm{C}$ NMR (DMSO- $\left.d_{6}\right): \delta 20.7,25.0,26.6,27.2,31.8,117.3,119.0,123.8,124.7,128.5$, 139.4, 142.6, 145.4, 156.3, 158.7. HRMS (ESI) calcd for $\mathrm{C}_{17} \mathrm{H}_{18} \mathrm{ClN}_{4} \mathrm{~S}[\mathrm{M}+\mathrm{H}]^{+} 345.0936$, found 345.0935 .

$\boldsymbol{N}^{\mathbf{2}}$-Benzyl-6,7-dihydro-5H-cyclopenta[b][1,3]thiazolo[4,5-e $]$ pyridine-2,8-diamine (4d0). Dark brownish solid, m.p. 143-145 ${ }^{\circ}$ C. IR: $3459(\mathrm{NH}), 3296$ and $3163\left(\mathrm{NH}_{2}\right), 2952\left(\mathrm{CH}_{2}\right), 1562$ $\left(\mathrm{NH}_{2}\right), 1531(\mathrm{NH}), 1348\left(\mathrm{NH}_{2}\right), 710$ and 697 (monosubstituted benzene) $\mathrm{cm}^{-1} .{ }^{1} \mathrm{H}$ NMR (DMSO$\left.d_{6}\right): \delta 1.8\left(\mathrm{~m}, 2 \mathrm{H}, \mathrm{CH}_{2}\right), 2.55\left(\mathrm{~m}, 4 \mathrm{H}, 2 \times \mathrm{CH}_{2}\right), 4.4\left(\mathrm{~d}, 2 \mathrm{H}, \mathrm{CH}_{2}\right), 5.5\left(\mathrm{~s}, 2 \mathrm{H}, \mathrm{NH}_{2}\right), 7.15(\mathrm{~m}, 5 \mathrm{H}$, monosubstituted benzene), $8.0(\mathrm{~s}, 1 \mathrm{H}, \mathrm{NH}) .{ }^{13} \mathrm{C}$ NMR (DMSO- $\left.d_{6}\right): \delta 22.7,27.2,33.5,46.5$, $116.3,126.9,127.6,128.2,130.8,139.2,140.6,151.1,158.5,161.4$. HRMS (ESI) calcd for $\mathrm{C}_{16} \mathrm{H}_{17} \mathrm{~N}_{4} \mathrm{~S}[\mathrm{M}+\mathrm{H}]^{+} 297.1168$, found 297.1178.

$\boldsymbol{N}^{\mathbf{2}}$-Benzyl-5,6,7,8-tetrahydro[1,3]thiazolo[5,4-b]quinoline-2,9-diamine (4d1). Beige solid, m.p. $140-142^{\circ} \mathrm{C}$. IR: $3401(\mathrm{NH}), 3339$ and $3121\left(\mathrm{NH}_{2}\right), 2919\left(\mathrm{CH}_{2}\right), 1599\left(\mathrm{NH}_{2}\right), 1548(\mathrm{NH})$, $1450\left(\mathrm{CH}_{2}\right), 1337\left(\mathrm{NH}_{2}\right), 737$ and 694 (monosubstituted benzene) $\mathrm{cm}^{-1} .{ }^{1} \mathrm{H}$ NMR (DMSO- $\left.d_{6}\right): \delta$ $1.7\left(\mathrm{~m}, 4 \mathrm{H}, 2 \times \mathrm{CH}_{2}\right), 2.5\left(\mathrm{~m}, 2 \mathrm{H}, \mathrm{CH}_{2}\right), 2.6\left(\mathrm{~m}, 2 \mathrm{H}, \mathrm{CH}_{2}\right), 4.6\left(\mathrm{~d}, 2 \mathrm{H}, \mathrm{CH}_{2}\right), 5.5\left(\mathrm{~s}, 2 \mathrm{H}, \mathrm{NH}_{2}\right)$, $7.3\left(\mathrm{~m}, 5 \mathrm{H}\right.$, monosubstituted benzene), $8.2(\mathrm{~s}, 1 \mathrm{H}, \mathrm{NH}) .{ }^{13} \mathrm{C}$ NMR (DMSO- $\left.d_{6}\right): \delta 22.2,22.7$, 23.1, 32.4, 46.5, 111.9, 126.9, 127.6, 128.3, 130.7, 139.2, 141.9, 149.7, 150.1, 161.4. HRMS (ESI) calcd for $\mathrm{C}_{17} \mathrm{H}_{19} \mathrm{~N}_{4} \mathrm{~S}[\mathrm{M}+\mathrm{H}]^{+} 311.1325$, found 311.1331 .

$N^{2}$-Benzyl-6,7,8,9-tetrahydro-5H-cyclohepta[b][1,3]thiazolo[4,5-e $]$ pyridine-2,10-diamine (4d2). Brownish solid, m.p. 198-200 ${ }^{\circ} \mathrm{C}$. IR: $3476(\mathrm{NH}), 3371$ and $3164\left(\mathrm{NH}_{2}\right), 2925\left(\mathrm{CH}_{2}\right), 1613$ $\left(\mathrm{NH}_{2}\right), 1451\left(\mathrm{CH}_{2}\right), 1345\left(\mathrm{NH}_{2}\right), 754$ and 697 (monosubstituted benzene) $\mathrm{cm}^{-1} .{ }^{1} \mathrm{H}$ NMR $\left(\mathrm{DMSO}-d_{6}\right): \delta 1.5\left(\mathrm{~m}, 4 \mathrm{H}, 2 \times \mathrm{CH}_{2}\right), 1.7\left(\mathrm{~m}, 2 \mathrm{H}, \mathrm{CH}_{2}\right), 2.6\left(\mathrm{~m}, 2 \mathrm{H}, \mathrm{CH}_{2}\right), 2.7\left(\mathrm{~m}, 2 \mathrm{H}, \mathrm{CH}_{2}\right), 4.5$ $\left(\mathrm{d}, 2 \mathrm{H}, \mathrm{CH}_{2}\right), 5.5 .\left(\mathrm{s}, 2 \mathrm{H}, \mathrm{NH}_{2}\right), 7.3\left(\mathrm{~m}, 5 \mathrm{H}\right.$, monosubstituted benzene), $8.2(\mathrm{~s}, 1 \mathrm{H}, \mathrm{NH}) .{ }^{13} \mathrm{C}$ NMR (DMSO- $\left.d_{6}\right): \delta 20.7,25.1,26.5,27.3,31.9,46.5,117.2,126.9,127.1,128.2,131.6,139.2$, 141.5, 148.2, 157.1, 161.9. HRMS (ESI) calcd for $\mathrm{C}_{18} \mathrm{H}_{21} \mathrm{~N}_{4} \mathrm{~S}[\mathrm{M}+\mathrm{H}]^{+}$325.1481, found 325.1490 . 


\section{Acknowledgements}

Authors thank the University Paul Verlaine-Metz (France) and the University of Luxembourg for supporting this work, Mrs Véronique Vaillant of the University Paul Verlaine-Metz for recording the NMR spectra and Dr Serge Schneider of the Laboratory of Toxicology of the Laboratoire National de Santé (Luxembourg) for recording the IR and MS spectra.

\section{References}

1. Thomae, D.; Kirsch G.; Seck, P. Synthesis 2007, 1027.

2. Seck, P.; Thomae, D.; Kirsch, G. J. Heterocyclic Chem. 2008, 45, 853.

3. Thomae, D.; Kirsch, G.; Seck, P. Poster presentation at the XXIIId European Colloquium on Heterocyclic Chemistry, Antwerp, September 9-13, 2008.

4. Thomae, D.; Kirsch, G.; Seck, P. Synthesis 2008, 1600.

5. Thomae, D.; Perspicace, E.; Hesse, S.; Kirsch, G.; Seck, P. Tetrahedron 2008, 64, 9309.

6. Gewald, K. (a) Z. Chem. 1961, 11, 349 (b) Gewald, K. Z. Chem. 1962, 2, 305 (c) Gewald, K.; Schinke, E.; Boettcher, H. Chem. Ber. 1966, 99, 94 (d) Gewald, K.; Schinke, E. Chem. Ber. 1966, 99, 271 (c) Sibor, J. ; Pazdera, P. Molecules 1996, 1, 157.

7. Freeman, F.; Kim, D.S.H.L. J. Org. Chem. 1991, 56, 4645.

8. Hirota, K.; Kazaoka, K.; Sajiki, H. Bioorg. Med. Chem. 2003, 11, 2715.

9. Marco, J.L.; de los Rios, C.; Garcia, A.G.; Villarroya, M.; Carreiras, M.C.; Martins, C.; Eleuterio, A.; Morreale, A.; Orozco, M.; Luque, F.J. Bioorg. Med. Chem. 2004, 12, 2199. 